Musées, Patrimoine et Culture scientifiques et techniques

$137 \mid 2011$

septembre - octobre 2011

\title{
Quand la technologie s'invite au musée
}

When 'technology' invites itself into museums

\section{Lucie Daignault et Claire Cousson}

URL : http://journals.openedition.org/ocim/950

DOI : 10.4000/ocim.950

ISSN : 2108-646X

Éditeur

OCIM

Édition imprimée

Date de publication : 1 septembre 2011

Pagination : 5-11

ISSN : 0994-1908

Référence électronique

Lucie Daignault et Claire Cousson, "Quand la technologie s'invite au musée », La Lettre de l'OCIM [En

ligne], 137 | 2011, mis en ligne le 01 septembre 2013, consulté le 04 mai 2019. URL : http://

journals.openedition.org/ocim/950; DOI : 10.4000/ocim.950 
milieu muséal et des projets comparables à celui de Copyright humain ont fleuri dans un grand nombre d'institutions muséales. On compte ainsi aujourd'hui plus d'une soixantaine d'applications iPhone développées dans les musées du monde entier, notamment en Amérique du Nord et en Europe (voir encadré p. 10).

Mais le multimédia est un média complexe dans une exposition, puisqu'il est régi par deux niveaux de scénarisation : le sien et celui de l'exposition. En outre, c'est un outil technologique récent qui nécessite un mode d'utilisation spécifique ${ }^{(5)}$.

Comment cette offre a-t-elle été reçue par les visiteurs de Copyright humain ? Quels usages en ont-ils fait et que dire de leur expérience de visite ? Et finalement quel est le rôle du support mobile dans une exposition comme Copyright humain?

\section{Copyright Humain : une exposition réflexion}

Copyright humain était une exposition réflexion invitant le public à suivre la plus irréductible faculté de l'humanité, la pensée. Elle se divisait en trois zones correspondant chacune à un âge de la pensée humaine.

La première zone, intitulée «Le pré-humain, la pensée fossile », explorait l'origine de la pensée humaine en remontant à la pensée fossile, celle des primates. Elle illustrait ainsi la proximité entre les grands singes et les humains, du point de vue comportemental et inné. Présentant une reconstruction de l'histoire évolutive des hominidés sous forme d'un arbre phylogénétique conçu comme une installation scénographique et associé à des objets archéologiques (les outils), elle indiquait les similitudes tout comme les différences accompagnant l'émergence d'une pensée qui intègre représentation et conceptualisation.

La deuxième zone de l'exposition, «Humain, un animal culturel », consacrée à l'âge de la pensée humaine, proposait d'entrée de jeu d'explorer la transmission du langage sous sa forme orale comme écrite. Elle montrait ensuite la capacité de l'homme à symboliser, à inventer, à développer une spiritualité et une créativité, donc une pensée culturelle et créatrice par laquelle il se distingue définitivement du règne animal. Elle s'intéressait enfin au cerveau, organe de la pensée au coeur des explorations des neurosciences, de la psychologie et de la philosophie. Les découvertes de ces disciplines étaient présentées, ainsi que la fragilité du cerveau et ses dysfonctions.

La dernière zone de l'exposition, "Post-humain, On ou Off ? » consacrée à l'âge posthumain, présentait diverses installations permettant d'illustrer le fait que le monde qui nous entoure est un pur produit de la raison et de la construction humaine. Elle évoquait également, avec l'œuvre Souls Awaiting A Future de John Feodorov, la transition entre l'humain d'aujourd'hui et celui transformé de

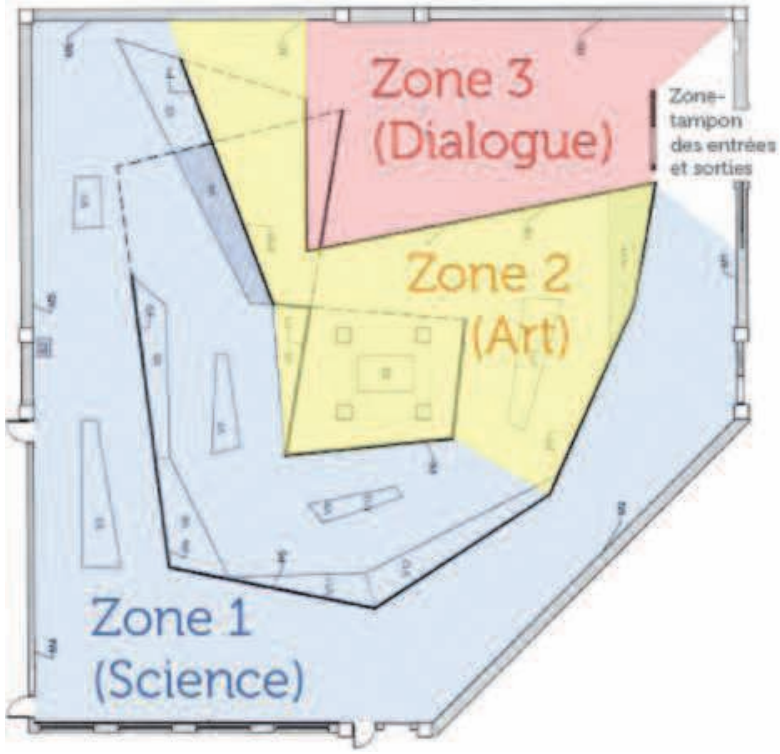

Une exposition divisée en trois zones (figure extraite de Pensée humaine. Scénario. Document interne. Québec, musée de la Civilisation, 2009). (c) L. Daignault

demain. Elle montrait aussi le partenariat de l'intelligence humaine avec des machines en traitant de l'intelligence artificielle. Enfin, le visiteur était invité à entrer dans la zone du « Dialogue artificiel », installation technologique qui l'amenait à écouter et à dialoguer avec des artistes, des scientifiques et des penseurs contemporains témoignant de leurs réflexions sur la pensée artificielle et ses possibles conséquences sur la pensée humaine.

\section{Une visite commentée avec un iPod Touch}

Deux types d'expérience étaient proposés aux visiteurs de l'exposition : la visite commentée avec un iPod Touch et la visite libre. La visite offerte avec l'appareil suivait la trame narrative de l'exposition. À certains moments, elle en résumait le propos en pointant ses objets phares, tandis qu'à d'autres moments, elle permettait d'aller plus loin. Au total, 24 stations ponctuaient le parcours des visiteurs. Le contenu présent dans l'application était varié (narrations, photographies, vidéos, entrevues). À ces contenus s'ajoutait aussi l'ensemble des cartels et des panneaux écrits qui accompagnaient les artefacts de l'exposition, de manière à solliciter, parfois en même temps, au même point de parcours, à la fois la lecture, la vision et l'audition. Il était également possible au visiteur de laisser des commentaires qui s'ajouteraient au contenu de l'appareil sur l'exposition.

Les visiteurs pouvaient louer un appareil (coût 2 dollars) à l'entrée de l'exposition, ou bien utiliser le leur (ou un téléphone cellulaire donnant accès à Internet) en accédant par téléchargement à l'application d'accompagnement de l'exposition. Les 
objectifs poursuivis par le musée à travers cette expérience de visite autonome de l'exposition à l'aide d'une technologie actuelle aux multiples possibilités multimédia étaient de créer un contexte pour une première expérimentation de ce type d'appareil et de ses possibilités, mais aussi de se positionner comme un musée innovateur aux niveaux des technologies, de la médiation et de la participation citoyenne ${ }^{(6)}$.

\section{Méthodologie de l'étude : une démarche qualitative}

Les partis pris de l'étude de Copyright humain menée par le service de la Recherche et de l'Évaluation du musée de la Civilisation sont étroitement liés au caractère expérimental du projet, à savoir l'utilisation d'un iPod Touch pour visiter l'exposition. Les questionnements à la base de l'évaluation ont été de deux ordres : en amont de l'utilisation du « guide multimédia » (les attentes exprimées, les motivations à une telle visite) ; et en aval, une fois l'outil en main (le profil des utilisateurs, la satisfaction par rapport à l'expérience de visite, le comportement de visite). Pour répondre à ces questionnements, une approche de nature qualitative a été privilégiée, qui s'imposait d'elle-même de par la nouveauté du sujet et le peu de recherches sur cette application dans les musées. L'intérêt de l'approche qualitative est qu'elle permet de saisir le sens donné aux pratiques de visite et l'interprétation de ces pratiques par les personnes interrogées ${ }^{7}$ ).

De juillet à septembre 2010, des entretiens semi-directifs ont ainsi été menés auprès de 31 personnes, à la sortie de l'exposition. De plus, même si le public-cible de cette nouvelle application technologique se limitait aux 18-34 ans, tous les groupes d'âge ont cependant été inclus dans l'étude, puisque cette dernière visait aussi à cerner le profil des utilisateurs de l'iPod Touch. Le schéma d'entrevue utilisé pour la conduite des entretiens a été élaboré à partir d'observations in situ réalisées au printemps 2010, et chaque entrevue a été transcrite verbatim. En outre, une évaluation a également été menée auprès de 44 personnes ayant visité l'exposition sans l'appareil, afin de pouvoir comparer les expériences de visite des utilisateurs et des non utilisateurs et de déterminer leurs différences de profils. Dans l'optique d'éclairer l'expérience de visite multimédia, seuls les résultats significatifs de cette comparaison sont présentés dans cet article.

\section{Le profil des utilisateurs}

La majorité des utilisateurs de l'iPod Touch dans Copyright humain se révèlent être des hommes (61 \%), contrairement à la visite de l'exposition sans l'appareil (les hommes ne constituent que $48 \%$ de l'échantillon). De plus, ce résultat diffère de la tendance générale de visite au musée de la Civilisation,

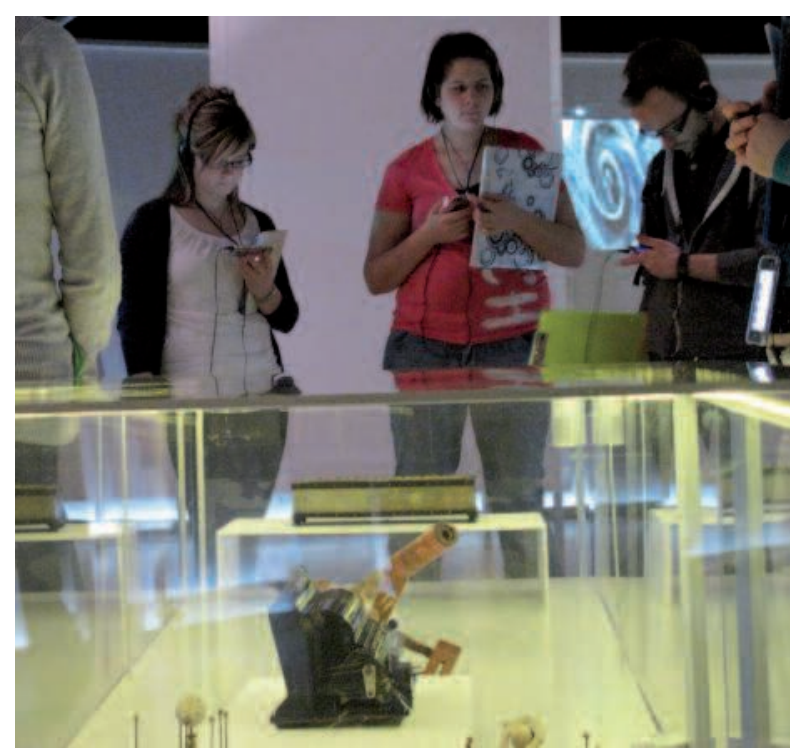

Des utilisateurs aux profils multiples

(c) Ana-Laura Baz

mais se trouve en concordance avec les résultats des principales enquêtes menées auprès d'utilisateurs de nouvelles technologies. Autre différence avec la fréquentation habituelle du musée, les visiteurs ayant un diplôme d'études secondaires ou collégiales comme dernier diplôme obtenu sont présents en plus grande proportion quà l'habitude. Cependant, les diplômés de l'enseignement supérieur se trouvent être les plus nombreux parmi les utilisateurs. De plus, les étudiants sont présents dans une proportion importante (32\%). En outre, bien que l'enquête se soit déroulée en pleine saison touristique, les trois-quarts des utilisateurs interrogés proviennent des villes de Montréal et de Québec.

\section{Profil des utilisateurs de l'iPod Touch dans Copyright humain}

\author{
Des hommes $(61 \%)$ \\ Tranches d'âge : 18-24 ans (36 \%) et 45-54 ans (32\%) \\ Des étudiants (32\%) \\ Des couples $(39 \%)$ et des familles $(32 \%)$ \\ Des diplômés de l'enseignement supérieur (64\%) et des visiteurs \\ familiers du musée de la Civilisation (61 \% ont déjà visité le musée) \\ Des visiteurs provenant de Montréal (45\%) et de Québec (32\%)
}

Tel que prévu par l'équipe de conception, le public jeune initialement ciblé par l'iPod Touch (les moins de 24 ans) s'est montré au rendez-vous (36\%), et particulièrement les jeunes de moins de 18 ans (23\%). Ce public est cependant suivi de près par les $45-54$ ans $(32 \%)$. Ce résultat qui peut paraître surprenant ne l'est point si l'on considère que plusieurs de ces visiteurs étaient accompagnés d'adolescents, lesquels ont manifestement fait pression auprès de leurs parents pour visiter avec un téléphone. En effet, le tiers des 
personnes interrogées étaient accompagnées d'enfant(s) de moins de 18 ans. De ce fait, les familles constituent une bonne proportion des visiteurs ayant utilisé l'iPod Touch, tandis qu'elles ne représentent que $17 \%$ des visiteurs libres. Il est possible qu'un sujet aussi abstrait que la pensée ait pu rebuter certaines familles avec enfants, qui se sont soit détournées de la visite, soit ont choisi d'utiliser l'appareil. Quant aux autres répondants, ils ont essentiellement visité en compagnie de leur conjoint(e) (39\%). Enfin, l'enquête a révélé que les personnes qui n'en étaient pas à leur première visite au musée de la Civilisation étaient les plus enclines à utiliser l'appareil (61\%), tandis que les primo-visiteurs constituent au contraire $52 \%$ des visiteurs libres interrogés.

\section{Le choix d'une visite avec l'iPod Touch : un outil anticipé comme un remplaçant de l'audioguide}

Seulement un répondant sur six avait prévu de faire la visite de Copyright humain avec un iPod Touch, en se renseignant préalablement sur le site Internet du musée. Ainsi, la majorité des utilisateurs ont décidé sur le coup de choisir l'appareil, notamment afin d'avoir le maximum d'informations possible sur l'exposition et de ne rien rater. Le choix de l'iPod est aussi anticipé par plusieurs comme un outil d'aide à la visite, qui serait en quelque sorte l'équivalent d'un audioguide, comme l'indique ce commentaire d'un visiteur : "Je m'attendais à ce que ce soit un peu comme les écouteurs qu'on a dans les autres endroits ». En associant l'appareil à un audioguide, les visiteurs s'attendent donc à des choses dites ou racontées, qu'elles soient de l'ordre du renseignement, de l'explication, de l'évocation ou de l'anecdote, un peu à la manière d'un

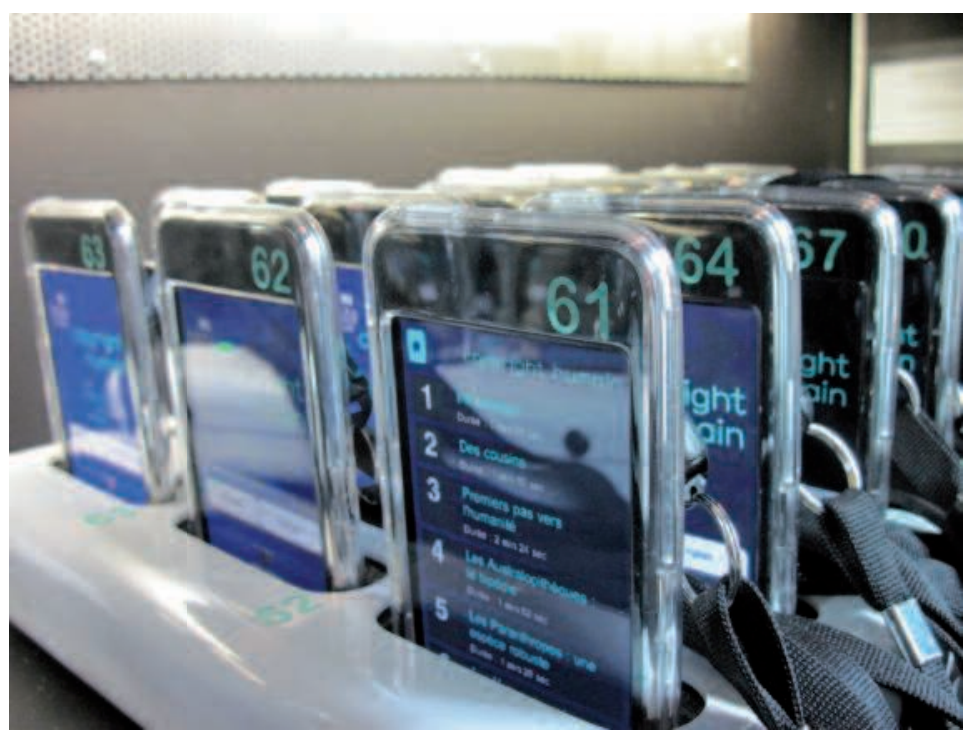

(C) L. Daignault guide animateur : "Je me disais que cela allait remplacer un guide. Habituellement, quand il y a un guide, je fais toujours la visite une première fois avec le guide, puis une seconde fois sans le guide, pour revoir ce que j'ai trouvé le plus intéressant. Et cette fois-ci, je me suis dit "il ne va pas y avoir de guide, il va y avoir l'iPod, alors on va utiliser l'iPod" ». L'autonomie offerte par un tel outil constitue un avantage certain pour les visiteurs qui souhaitent être guidés tout en gardant leur liberté. Ils n'ont ainsi pas à subir les contraintes du groupe (horaire, choix des objets et rythme imposés), l'outil autorisant au contraire la liberté des pratiques individuelles (Deshayes) : « L'iPod, je pense que j'aime mieux ça, parce que ça nous permet de lire à notre rythme, tandis qu'avec le guide, il faut attendre là, se déplacer en masse, ce n'est pas plaisant parce qu'on ne peut pas toujours voir, quand il y a beaucoup de monde, on ne peut pas voir, on est en arrière de 10-12 personnes... ». Le support mobile comme l'audioguide ou le téléphone permet finalement de « concilier le besoin contradictoire d'assistance et d'autonomie» (8).

Autre motivation à utiliser un outil multimédia pour visiter l'exposition, plusieurs des personnes interrogées avouent avoir une préférence prononcée pour l'écoute plutôt que pour la lecture. L'appareil, qui mettait à la disposition des visiteurs des choses dites et agissait comme un complément, voire un remplaçant aux textes de l'exposition, constituait alors un outil pertinent pour ces visiteurs. De plus, selon Sophie Deshayes, "une des motivations principales des utilisateurs [des supports mobiles] est l'opportunité d'écouter et de regarder en même temps, le support résolvant en cela la difficulté ressentie par rapport au positionnement de certains textes ou cartels (on doit lire et aller voir ensuite... ) » (9). La mobilité procure en effet «le bénéfice de la simultanéité $d u$ regard et de l'accès aux éléments de médiation » (10). C'est d'ailleurs dans ce sens-là que plusieurs répondants ont expliqué leur choix d'utiliser le téléphone : "Je préfère écouter l'audioguide que lire les explications. Cela donne plus de chance par exemple de voir ce qui est exposé ».

Enfin, la technologie en tant que telle offre aussi sa part d'intérêt et est considérée par les parents accompagnés d'adolescents comme un incitatif à la visite : "J'ai choisi l'iPod parce que j'étais avec ma fille, et cela va l'attirer plus au niveau technologie. Et aussi pour avoir toute l'information, la narration était intéressante, mais la principale raison, c'est pour inciter ma fille à venir voir cette exposition-là ».

Quant aux visiteurs libres interrogés sur leurs raisons de ne pas choisir l'appareil, ils ont principalement mentionné leur manque d'intérêt vis-à-vis de l'appareil sans voir ce qu'il pouvait leur apporter, accordant leur préférence à la lecture et à l'observation des objets, en d'autres termes à 
une visite classique : "Et bien moi je n'avais pas d'intérêt à l'écouter en plus. Ce n'est pas comme si c'était une exposition sur la musique, et que cela ajoutait quelque chose de plus. Là on est capable de lire puisqu'on imagine que c'était les mêmes informations ».

\section{Des comportements de visite assez diversifiés}

La visite avec l'outil multimédia a été variée en fonction des utilisateurs, et la gestion de la concurrence entre l'appareil et les médias présents dans l'exposition (bornes vidéo et textes imprimés) s'est avérée très hétérogène. Si près de la moitié des utilisateurs interrogés ont intégralement écouté les narrations de l'appareil et ont ainsi donné la priorité à l'iPod Touch, pensant que celui-ci contenait l'essentiel du contenu de l'exposition, d'autres ont davantage alterné entre l'écoute des narrations, la lecture des textes et l'observation des vidéos de l'exposition : «Encore là, j’ai fait confiance au concepteur. S'il l'a mis sur l'iPod, c'est que cela doit être les points majeurs. Donc le texte, j'aurais pu prendre le temps de le lire, j'aurais pu prendre le temps de regarder toutes les vidéos, mais je ne l'ai pas fait ».

D'autres répondants, qui ont constaté une certaine redondance entre les narrations, les textes de l'exposition et les vidéos, ont eux aussi privilégié l'appareil en pensant obtenir la même information : "À un moment donné je lisais le texte pendant qu'elle parlait, et puis c'était presque la même chose... Du coup je me suis dit: qu'est-ce que je fais, j'écoute ou je lis?».

Certaines personnes ont plutôt choisi d'alterner l'écoute des narrations et la lecture des textes pour diversifier leurs expériences de visite et pour augmenter la rétention des contenus, se disant plus concentrées lors de la lecture que de l'écoute : "J'avais l'iPod qui me guidait, c'est lui qui me guidait le plus, puis je revenais an texte pour voir, parce que j'oubliais des

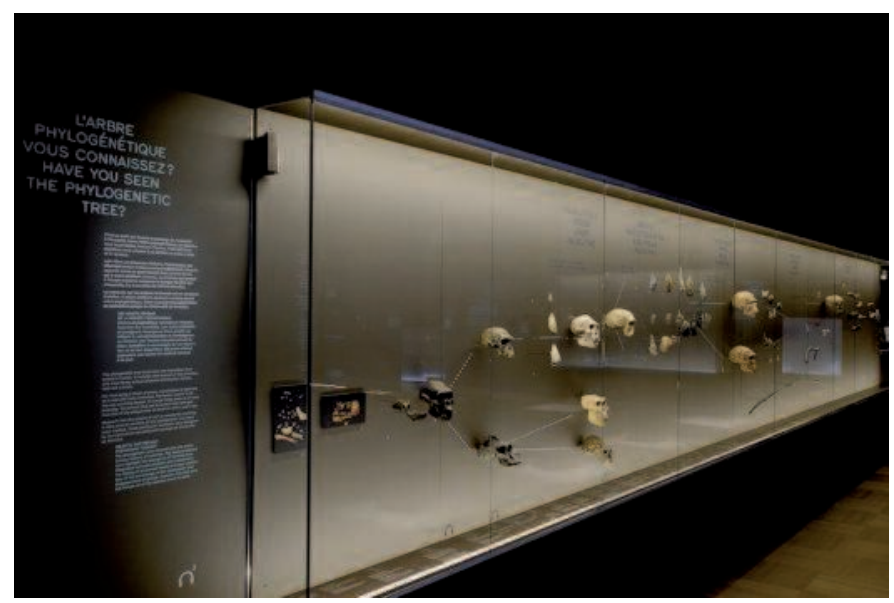

Des stations d'écoute de l'appareil signalées par un pictogramme qui prête à confusion. (C) Perspective/Amélie Breton choses qui avaient été dites sur l'iPod, alors je lisais. Donc c'était toujours l'iPod en premier, puis j'allais relire les textes qui m'intéressaient vraiment, et les autres je les lisais partiellement».

Par ailleurs, la comparaison effectuée entre les utilisateurs et les non utilisateurs a montré que le parcours induit par l'outil multimédia différait du parcours libre. En effet, l'utilisation de l'appareil, qui proposait 24 stations dans l'exposition, faisait en sorte que le visiteur optait pour un parcours logique essentiellement structuré par les stations d'écoute suggérées, alors que les visiteurs en situation de visite libre interrogés ont plutôt emprunté un parcours lié à une curiosité intuitive et à leur intérêt.

\section{Une visite allongée et enrichie grâce à l'iPod}

La durée de visite avec l'appareil a été modifiée par rapport à une visite traditionnelle, et cela pour l'ensemble des utilisateurs. En effet, pour la majorité d'entre eux, leur visite a été allongée du fait de l'iPod, comme en témoigne ce commentaire : "Je trouve que cela a modifié ma visite, parce que je ne suis pas un gros lecteur, et puis j'aurais pas passé autant de temps dans l'exposition. En entendant je trouve que cela passe mieux le temps qu'en lisant». Plus précisément, $50 \%$ des visiteurs ont déambulé au moins une heure dans l'exposition, comparativement à $11 \%$ pour les visiteurs autonomes.

\begin{tabular}{|l|c|c|}
\hline $\begin{array}{l}\text { Durée } \\
\text { de la visite }\end{array}$ & $\begin{array}{c}\text { Avec } \\
\text { l'iPod Touch }\end{array}$ & $\begin{array}{c}\text { Sans } \\
\text { l'iPod Touch }\end{array}$ \\
\hline Moins de 15 minutes & $3 \%$ & $8 \%$ \\
$15-29$ minutes & $7 \%$ & $45 \%$ \\
$30-44$ minutes & $20 \%$ & $26 \%$ \\
$45-59$ minutes & $20 \%$ & $11 \%$ \\
1 heure & $27 \%$ & $6 \%$ \\
Plus de l heure & $23 \%$ & $5 \%$ \\
\hline
\end{tabular}

La visite a aussi été jugée plus intéressante, plus compréhensible et plus riche avec l'outil multimédia, tant au niveau des informations reçues que de la forme plus interactive, agréable et autonome qu'elle a prise : «Cela mia aidé à mieux comprendre, cela facilitait les explications... Avec l'iPod Touch, je n'ai pas vraiment eu à me poser de questions, parce qu'il répondait quand je n'étais pas certain d'une information».

\section{Une satisfaction unanime, atténuée par quelques critiques}

Au final, la totalité des répondants a manifesté sa satisfaction à l'égard de l'appareil. Ses points forts relevés par les utilisateurs sont nombreux et concernent aussi bien l'autonomie de visite et l'interactivité qu'il procure, que les 


\section{Un état des lieux de l'utillisation de la fechnologie 3G au musée}

Si l'expérience du musée de la Civilisation constitue une première au Québec, qu'en est-il ailleurs, dans les autres musées au Canada et dans le monde ? Nous présentons cidessous quelques exemples afin d'illustrer la diversité existante dans l'utilisation de ces nouvelles technologies et de dresser un état des lieux (non exhaustif) de la question.

Le musée de Cluny en France (musée du Moyen Âge) est un pionnier en Europe puisqu'il lance dès mars 2009 une application iPhone qui permet d'effectuer une visite commentée à partir de son téléphone mobile. Le musée du Louvre lui emboîte le pas en avril 2009, à l'occasion des Nocturnes Jeunes, activité qui donne l'opportunité aux jeunes de découvrir tous les vendredis soirs Le Louvre différemment. Le Louvre propose alors à ses jeunes visiteurs d'emprunter un iPhone pour réaliser une visite originale avec une médiation intégrée. Il s'agit de plusieurs parcours différenciés imaginés en partenariat avec un artiste, un philosophe ou encore un historien, qui permettent de découvrir des œuvres au travers d'un regard singulier. Le visiteur a en outre la possibilité de créer et de proposer son propre parcours, qui pourra être suivi par les prochains utilisateurs (1). Le musée du Louvre développe également dans la foulée, en novembre 2009, une application bilingue permettant de découvrir les plus belles œuvres du musée grâce à des commentaires, des zooms et des vidéos (2).
La National Gallery de Londres entreprend une démarche similaire dès juin 2009 pour présenter de manière novatrice ses collections aux visiteurs. C'est en effet le premier grand musée d'art du monde à lancer une application iPhone appelée Love Art. Cette application permet à son utilisateur de découvrir 250 peintures parmi les plus fameuses de la collection du musée et d'avoir accès à plus de 200 minutes de contenu audio et vidéo ${ }^{(3)}$.

En Amérique du Nord, nombre de musées américains se sont également engagés dans la voie des plus récentes technologies. Le Brooklyn Museum a par exemple depuis avril 2009 une application qui agit comme un guide dans le musée, donnant accès au catalogue virtuel de l'institution. De même, le American Museum of Natural History a lancé en juillet 2010 une application permettant au visiteur de s'orienter dans le musée et d'avoir le choix entre suivre une variété de visites guidées conçues par le musée, ou bien créer son propre parcours à partir d'une liste d'artefacts exposés parmi les plus populaires.

Au Canada, c'est le musée canadien des Civilisations (MCC) de Gatineau qui a été la première institution à introduire une application iPhone en décembre 2009. Cette application permet aux visiteurs d'avoir accès via leur appareil aux audioguides de la salle du Canada et des Premiers Peuples, ainsi qu'à d'autres sources de renseignements sur le MCC (plan du musée, programmation, horaires, tarifs...) (4). informations complémentaires qu'il apporte, les présentations visuelles qui ont permis de mieux expliquer des points de l'exposition, ainsi que la sonnerie agissant à l'instar d'un guide et indiquant quand regarder l'écran. Les utilisateurs ont aussi conseillé d'exploiter encore davantage le potentiel de l'appareil, et notamment son écran, afin de véritablement le différencier de l'audioguide et d'apporter un vrai plus. Ils ont également encouragé le musée de la Civilisation à étendre l'expérience à toutes ses expositions, ce qui témoigne sans l'ombre d'un doute de leur enthousiasme et de leur intérêt pour cette technologie et son application au musée.

Cependant, quelques critiques ont été apportées. Par exemple, certains visiteurs peu familiers de cette technologie auraient apprécié avoir une meilleure formation sur l'utilisation de l'appareil avant d'entreprendre leur visite, afin de profiter pleinement de l'outil offert. De plus, l'écoute des vidéos été perturbée par des incompréhensions engendrées par la présence de l'appareil. En effet, certains visiteurs ont pensé que les vidéos de l'outil multimédia et celles de l'exposition étaient les mêmes, et se sont de fait concentrés sur l'appareil. En outre, le fait de devoir changer d'écouteurs entre le support mobile et les vidéos de la salle en a aussi découragé quelques-uns. Certaines personnes en visite autonome n’ont quant à elles pas écouté de vidéos dans l'exposition, car elles pensaient qu'elles n'étaient accessibles qu'aux visiteurs munis d'un iPod Touch. Le pictogramme illustrant des écouteurs et servant à indiquer les différentes stations d'écoute de l'appareil constitue la principale source de confusion et explique en partie ces incompréhensions.

\section{Que retenir de l'expérience?}

L'utilisation de la technologie $3 \mathrm{G}$ et de la téléphonie intelligente pour accompagner la visite de l'exposition Copyright humain a constitué une première réussie au musée de la Civilisation. L'engouement entourant les technologies des télécommunications ne touche pas seulement le milieu muséal québécois et des projets similaires ne cessent de se développer depuis quelques années dans un grand nombre d'institutions muséales, partout dans le monde. La variété et la multiplicité des applications iPhone développées par 
les musées dans une période relativement courte donnent la mesure du développement de ce phénomène. Elles confirment aussi la pertinence, voire la nécessité, de mettre en œuvre des projets intégrant une dimension technologique importante, tout en s'attachant à fournir des contenus de qualité, rappelant ainsi que la technologie n'est pas une fin en soi mais un outil au service d'une médiation avec le visiteur.

En effet, les ressources mises à la disposition des visiteurs n'ont de sens que si elles offrent des choix multiples qui ont été préalablement pensés pour proposer au visiteur une sélection de contenus balisés par une logique, à la fois celle de l'exposition et celle du scénario du support mobile. L'offre du multimédia mobile doit ainsi être complémentaire aux différentes sollicitations déjà présentes dans l'exposition, sans quoi son usage détourne plus qu'il n'accompagne, puisqu'il devient un énième choix possible concurrençant la visite elle-même (11). À cet égard, le recours à l'image via l'écran du support mobile est à utiliser avec discernement, les images ne devant pas se substituer à la contemplation des objets ou aux autres dispositifs muséographiques. De plus, les visiteurs devraient être avisés de manière très claire de la présence de différents médias dans l'exposition au préalable à leur visite, ainsi que de la redondance ou non de l'information entre les différents supports (textes, objets, vidéos, support mobile) afin d'éviter toute confusion et perte d'information.

Lors du développement d'un support mobile, les aspects suivants devraient donc être pris en considération : le mode de navigation, le mode de fonctionnement qui convoque l'aspect technique, et finalement le contenu non seulement du multimédia mais aussi de l'exposition, afin d'établir la relation entre les deux et de déterminer leurs rôles respectifs.

\section{Notes}

(1) Voir le site de la compagnie Musetrek, qui a développé ce produit : http://cite.musetrek.com

(2) Musée du Louvre : www.louvre.fr

(3) National Gallery : www.nationalgallery.org.uk

(4) Musée canadien des civilisations : www.civilization.ca/mcc/accueil/ mcc-accueil

(5) Jovet, V. Le multimédia dans l'exposition : la double problématique de l'appropriation et de l'intégration d'un média marginal, Les institutions culturelles et le numérique, ICHIM 03, Paris, actes publiés avec le soutien de la Mission de la Recherche et de la Technologie du Ministère de la Culture et de la Communication, 2003, p. 2.

(6) Baz, A.-L. Une touche de pensée. Visiter l'exposition Copyright humain avec un iPod Touch. Document interne. Québec : Musée de la Civilisation, service de l'Éducation, 2009.
(7) Habib, M.-C. Sur quelques questions de méthode à propos des études qualitatives des publics de la Cité des Sciences et de l'Industrie, in Donnat, O. et Octobre, S. (dir.), Les publics des équipements culturels. Méthodes et résultats d'enquête. Paris : Ministère de la Culture et de la Communications, 2001, p. 129.

(8) Deshayes, S. L'usage des supports mobiles au musée, des audioguides classiques au multimédia nomade, Patrimoine \& Culture numérique, ICHIM 04, Paris, actes publiés avec le soutien de la mission de la Recherche et de la Technologie du ministère de la Culture et de la Communication, 2004, p. 9.

(9) Ibid., p. 7.

(10) Ibid., p. 10.

(11) Ibid., p. 13

\section{Bibliographie}

Davallon, J. L'exposition à l'œuvre : stratégies de communication et médiation symbolique. Paris : L'Harmattan, 2000, 378 p.

Deshayes, S. Interprétations du statut d'un audioguide, Études et communications, n²4, 2001, pp. 71-89.

Deshayes, S. Audioguides et musées, La Lettre de l'OCIM, nº79, 2002, pp. 24-31.

Deshayes, S. Une nouvelle génération d'audioguide : démarche de conception et choix du multimédia mobile au Museon Arlaten, La Lettre de l'OCIM, n 92,2004 , pp. 17-23.

Le Marec, J. L'usage en son contexte : sur les usages des interactifs et des cédéroms de musées, Réseaux, n¹01, 2000, pp. 173-196.

Tallon, L. et Walker, K. Digital technologies and the museum experience: handheld guides and other media. Lanham : AltaMira Press, 2008, 238 p.

Vidal, G. Contribution à l'étude de l'interactivité : les usages de multimédia de musée. Pessac : Presses universitaires de Bordeaux, 2006, 168 p.

Sites Internet :

- Musée de la Civilisation, exposition Copyright humain : www.mcq.org/ copyrighthumain/fr/

- Culture mobile : www.culturemobile.net

- Blog Muséomobile, l'actualité des technologies mobiles au musée : http://museomobile.wordpress.com/12 